\title{
Research on Exploration and Analysis of Supply Chain Flexibility Measure Index
}

\author{
$\mathrm{He} \mathrm{Tao}^{1 *}$, Liang Zhidong ${ }^{2}$, Ye Xinquan ${ }^{3}$ and Pang Jihong ${ }^{3}$ \\ ${ }^{1}$ Oujiang College of Wenzhou University, Wenzhou 325035 \\ ${ }^{2}$ School of management, Shangdong Youth University of Political Science. Jinan \\ 250103 \\ ${ }^{3}$ College of Mechanical \& Electrical Engineering, Wenzhou University \\ landyhe1010@163.com
}

\begin{abstract}
The supply chain risk assessment and management system has been developed, people still do not determine the way to overcome all these risks, especially for high impact, low probability events, such as SARS and the outbreak of foot-and-mouth disease, or a centralized terrorist attacks. This is because the lack of historical data to make all kinds of prediction and statistical tools fail. The modern supply chain were more vulnerable to risk, supply chain risk mainly comes from many aspects of the internal and external environment, the supply chain industry products background, upstream and downstream supply chain relationship, decision support. In this paper, the establishment of a complete supply chain model with variable structure, through the simulation method for quantitative observation of supply chain flexibility curve, and abstract to describe the elastic curve of a set of parameters; after the design of simulation experiment to observe the different supply chain structure parameters (inventory level, supply chain, supplier selection for number of sources) influence supply chain resilience curve.
\end{abstract}

Keywords: Supply chain; risk; information sharing; principal agent; infrastructure

\section{Introduction}

The stability of the supply chain is relative, the external environment and demand change is the norm, with the economic globalization, more and more uncertainty, reliability of supply chain become more and more weak, strengthen the key supply chain flexibility is to strengthen supply chain management change period of [1]. Guang Shi V [2] proposed three criteria for efficient supply chain flexibility: first, the strategic alliance of supply chain node enterprises, realize the maximization of the node enterprises to maximize their own interests and supply chain performance; second, to changes in the market response capacity and ability to adapt to the strategic change; third, the reaction speed to respond to emergencies [3].

The supply chain industry risk refers to the influence caused by uncertain market, industry and new products of the three and risk. Between the new product market and industry, not adapt or uncoordinated, uncertainty or change of customer demands, or deliberate pursuit of innovation and leading to make the right judgments suppliers not in the customer's final demand, will be on the supply chain of the industry caused by the impact and risk [4-6].

The standardization process refers to the similar function of factories and workers in the production process of standardization in the use of standard parts with, and can be interchanged in different products. The interchangeable parts, production equipment and workers allows a company to react quickly in the event of interruption, the allocation of

* Corresponding Author 
resources to go where they are most needed [7]. Like in its semi finished products factory in full use of equipment layout and production program for the same. The design and production of benefit from this standard, when the demand changes Intel can arbitrary allocation of production tasks in different factories.

The use of concurrent engineering in the key field of product development, production and sales can accelerate enterprise after the interruption of the recovery process, and improve the speed of response to market demand. Lucent technology, through the various functions (including engineering company, sales) centralized management form of concurrent engineering. Can very easily by this means the company observing all areas of work, and in times of emergency for various activities to make quick judgments. The current research field of supply chain flexibility is qualitative. Experts through interviews, the survey on a large number of enterprises, summarizes the types, supply chain risk serious harm, supply chain, elastic recovery phenomena in the process of supply chain flexibility enhancement of effective measures, so as to give the empirical regularity conclusions and recommendations, for enterprises to adopt.

\section{The Research Status of Supply Chain}

\subsection{Research on Supply Chain Risk Abroad}

Study on the elasticity of supply chain abroad, in 2001 only gradually taking shape, was launched from the angle of enhancing supply chain flexibility and. Muckstadt [11] that improving supply chain resilience by strengthening the cooperation between enterprises and try to eliminate the uncertainty of business environment to achieve, Christopher [12] advocates to build rapid response emergency supply network is an effective measure to obtain the supply chain flexibility, Rice [13] that adopts mixed flexible and redundant measures are relatively inventory good ways to enhance supply chain flexibility; Yossi Sheffi [14] in the Harvard Business Review also wrote that increase business redundancy, improve supply chain flexibility and create a risk oriented corporate culture is to enhance the three basic ways, supply chain flexibility and supply chain flexibility, improve and build the risk of enterprise culture is to increase the elasticity of the key, because increased redundancy will affect the utility.

\subsection{Research on Supply Chain Risk Domestic}

In China, the understanding of the connotation of supply chain flexibility is not the same. Liu Haohua [7] will supply chain flexibility is defined as: supply chain network system after the interrupt event occurs rapidly back to its original state or changes to the ability of the ideal state. Yi Haiyan et. al., [8] that the elasticity of supply chain refers to the whole supply chain adaptation to changes in the environment, including the flexibility and agility. Flexibility refers to the supply chain with the change of the market environment and conditions and adjusted in time to meet customer demand flexibility; agility the ability to make quick response to supply or demand unpredictable changes. Tan Ying [9-10] research that the nature of supply chain flexibility: maximum precision to meet customer demand for the quantity and diversity, on-demand production, much more, to small, no more and no less, and freely.

\section{The Model of Supply Chain Concept}

\subsection{Assembler Operation Mechanism}

Assuming that the assembly each finished piece need time hours, each production preparation time is 1 hours. Considering the assembly time can not be more than a day 
(second days will start generating assembly new), it is ultimately the implementation of assembly part number A:

$A_{A}= \begin{cases}S_{A}-I_{A}^{\prime} & \text { if } I_{A}^{\prime}<s_{A} \\ 0 & \text { if } I_{A}^{\prime}<s_{A}\end{cases}$

In it: $I_{A}^{\prime}=I_{A_{-}} I_{b a c k l o g}$

Risk before the influence of average waiting time and the initial value than

$y_{\text {wait_before }}=1.4-0.73^{x_{s}}+0.089^{x_{s}{ }^{2}}$

The main effect of average waiting time and the initial value:

$y_{\text {disrupptia_major }}=0.78+0.27^{x_{s_{-}}-0.061^{x_{s}}}$

$\frac{d y_{\text {disruption_major }}}{d x_{s}}=0.27-0.122 x_{s}$

The residual effects of the average waiting time and the initial value:

$y_{\text {disrupptio_after }}=1.1+0.13^{x_{s}-0.045^{x_{s}}}$

$\frac{d y_{\text {disruption_after }}}{d x_{s}}=0.13-0.09 x_{s}$

The main effect of waiting time and the initial value than the amplification

$y_{\text {amplificaion_major }}=-9.0+4.1 d_{-0.29} d^{2}$

$\frac{d y_{\text {amplificaion_major }}}{d d}=4.1-0.58 d$

$d_{0=7}$

Figure 1 depicts an enterprise in the supply chain after the interruption performance recovery. 


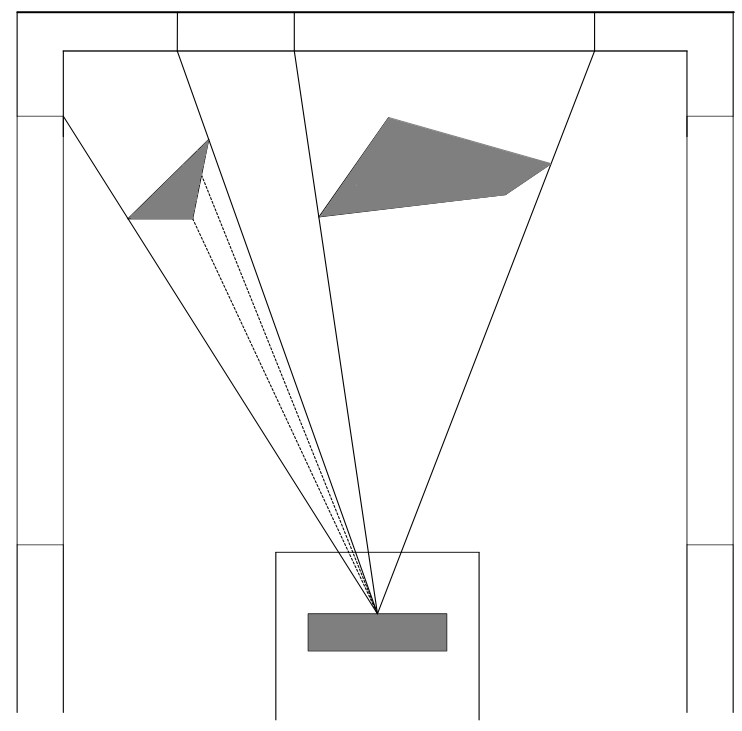

\section{Figure 1. Enterprise in the Supply Chain after the Interruption Performance Recovery}

\subsection{The Lower Reaches of the Supply Chain Risk}

On the supply chain risk relationship, because the trust relationship is between each other under the supply chain, once the relationship between change for the whole supply chain will be seriously impaired. If the inventory risk, when problems occur in sporadic supply chain process problems or inventory system, will cause damage to the supply chain; risk of procurement, procurement costs are too high, it will reduce the competitiveness of its products, to meet the demands of customers; distribution of risk occurrence, accidental transport failure or other human error, so that to improve the out of stock rate, increase the distribution cost and risk. A basic measure of supply chain risk management was shown in Figure 2.

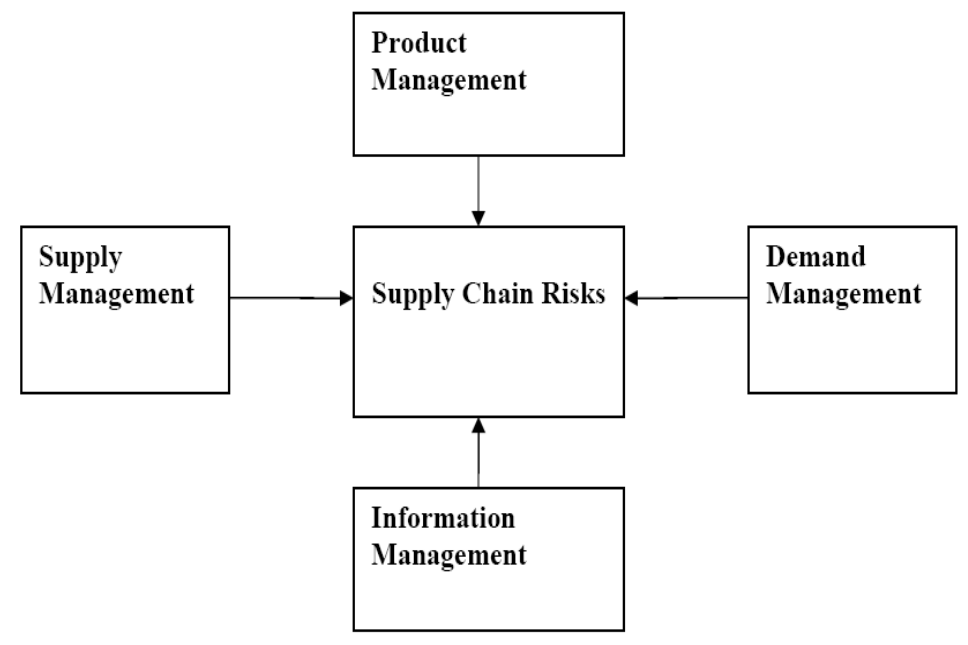

Figure 2. Basic Measures of Supply Chain Risk Management

Decision support risk from policy changes in the external environment, such as the national restrictions on free trade, affect significantly changes the international taxation and foreign exchange rate changes and controls for each link of the supply chain and the risk caused by. Policy support risk related to supply chain enterprises, more difficult to 
control or avoid, the most appropriate way is to establish emergency mechanism related to construction, flexible supply chain, to adapt to this change through their own adjustment.

\subsection{Supply Chain Information Sharing Mechanism}

The core enterprise in supply chain information sharing mechanism of the core enterprise based on refers to, is at the core position play a leading role in the supply chain alliance, in logistics, information flow, capital flow, technology, management coordination, strength is very strong and cohesive enterprise, around the core enterprise construction supply chain, not just from a supply and demand the supplier's supplier to the customer the customer simply

The value of existence of the core enterprise is that it is the promoter and ensure the efficient operation of the supply chain, it can make the whole supply chain always keep improving the pressure strong, how its function effect, directly affect the coordination and the efficiency of the supply chain.

\section{Experimental Results}

\subsection{Retailers Operating Mechanism}

When the goods arrived from assemblers supply retailers, this assumes all available goods are used immediately directly meet the unfinished customer orders. At the same time retailers record the supplier (superior assemblers) order time to complete, and update the number of less good. At the same time, assuming the outstanding orders at this time would continue to meet customer part if not completed orders. Suppose a customer and retail commercial very close, pick up don't need time. In order to calculate the customer waiting time $\mathrm{T}$, must be in the customer in the unfinished keep two attributes while waiting for the order queue: demand generation time and the current backlog size.

Two flow in any supply chain are in the opposite direction: from the customer to the retailer to assembly business until the manufacturer information flow (demand and order), and from the manufacturer to the logistics of assemblers to retailer and customer.

Because of this unit is a passive data unit (passive data units), then the simulation method of the simplest is to use data domain (data fields). Anylogic data definition language is Java, so we need to create three class Java (Java classes):

\section{demandSizeProbability - Table Function}

\begin{tabular}{|c|c|c|c|}
\hline Name: & demandSizeProbability & \multicolumn{2}{|l|}{, } \\
\hline \multicolumn{2}{|c|}{ Interpolation: } & \multicolumn{2}{|l|}{ Table Data: } \\
\hline None & $v$ & Argument & Function \\
\hline \multicolumn{2}{|c|}{ Out of Range: } & 1.0 & 0.2 \\
\hline Error & $v$ & 2.0 & 0.4 \\
\hline \multicolumn{2}{|l|}{ Value: } & 3.0 & 0.2 \\
\hline \multirow[t]{2}{*}{0.0} & & 4.0 & 0.1 \\
\hline & & 5.0 & 0.1 \\
\hline
\end{tabular}


International Journal of $u-$ and e- Service, Science and Technology

Vol. 9, No. 11 (2016)

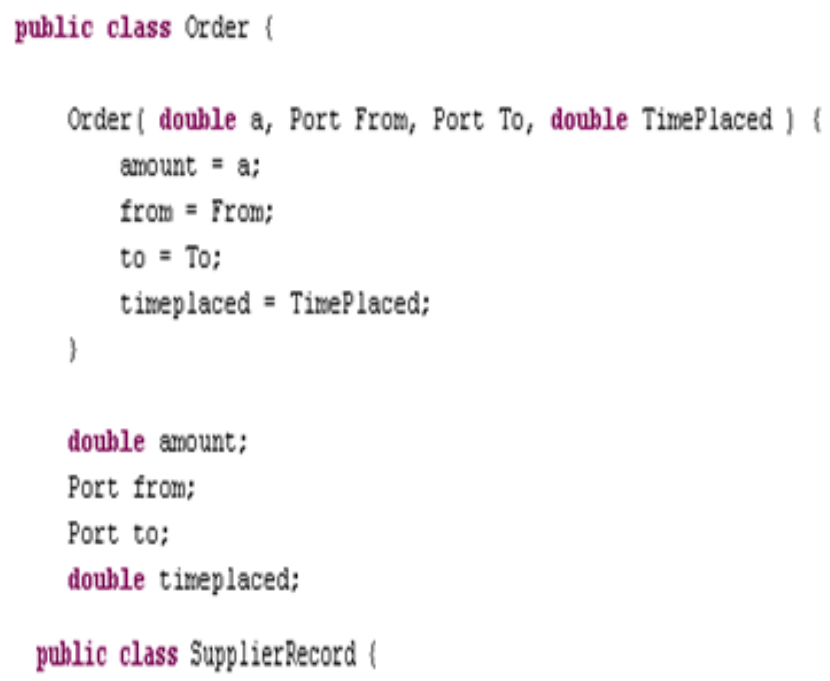




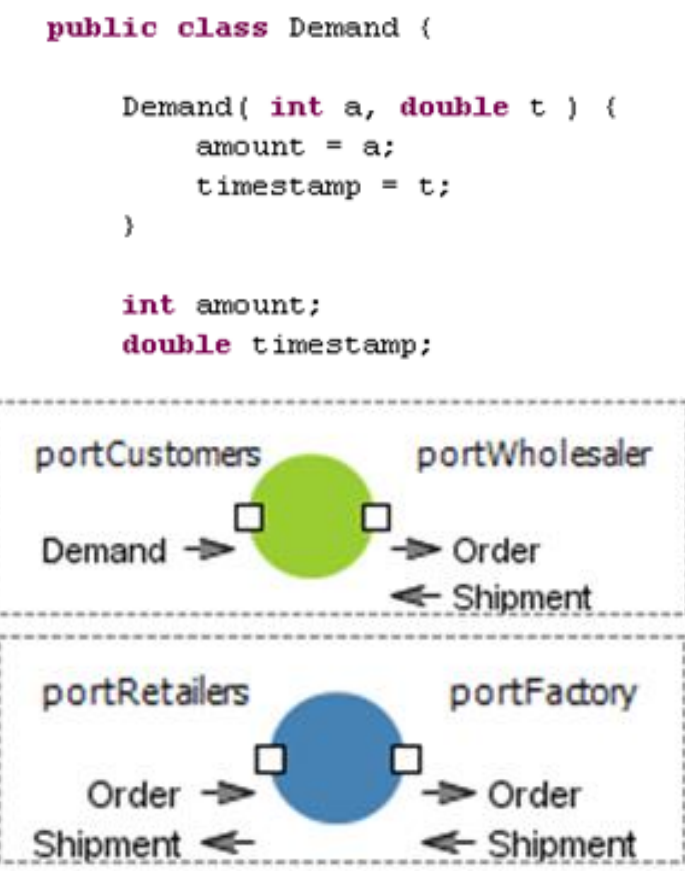

\section{Figure 3. Interface for Information Java Class Declaration}

We also need the Java class interface declarations for each object class is the interface to receive and send information: this statement can be used as to ensure that the sending and receiving proper inspection, also can simplify the process of information transfer Figure 4. In the design of the model, we ensure that the interaction form between objects only information transmission. This will ensure that the retailer and manufacturer, assembly independently, and model structure adjusted.

\subsection{Assembly Business Model}

We need to express the inventory level, record not complete orders. Because there is no difference between the same kinds of products in the supply chain, can use ordinary integer variables (plain integer variable) said the inventory level. Since the order processing using FIF principle, we can be in the collection list (LinkedList) record them. Plain variable and collection are the graphical representation in Anylogic (Figure 4):

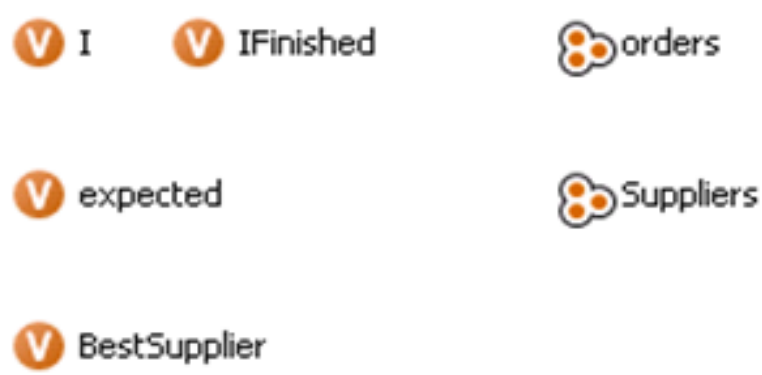

\section{Figure 4. Variable and Collection Icon}

The influence of the risk before the average waiting time, the main effect of the average waiting time, the residual effects of the average waiting time, average waiting time after the effect of the four parameters to describe the performance of supply chain is 
the change in absolute volume. The main effect of waiting time amplification, residual effect of waiting time amplification of these two parameters are used to describe the supply chain performance for interrupt sensitivity.

Another set of parameters are obtained to describe the elastic curve of supply chain supply capacity of anticlimax can be used the same method: risk before the influence of average waiting time, side effect of the average waiting time, the main effect of the average waiting time, the average waiting time for after effects, side effects of waiting time amplification, the main effect of waiting time amplification, side effects start time lag, the main effect of start time lag, lag time end effect. The output file into the MINITAB statistics software rendered the customer waiting time column chart Figure 5.

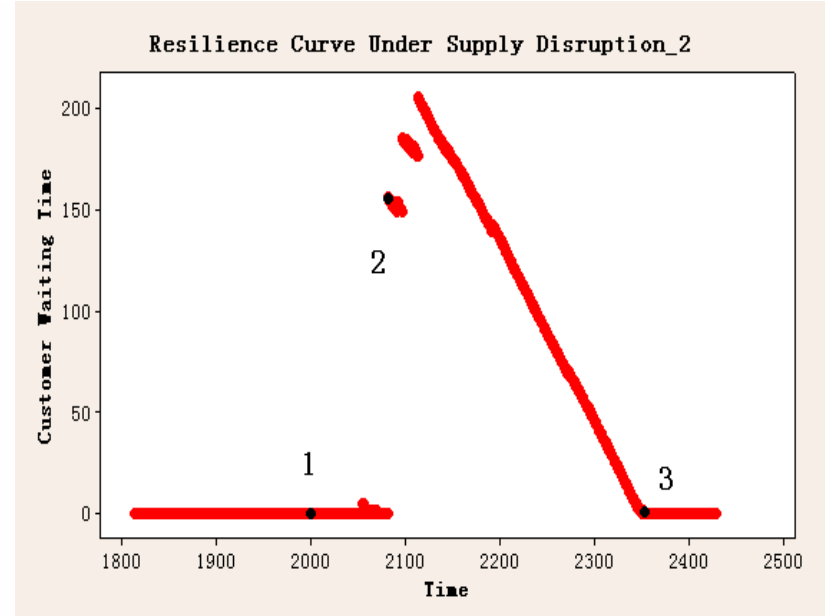

Figure 5. The Output File into the MINITAB Statistics Software Rendered the Customer Waiting Time Column Chart

The normalized data table was shown in Table 1, the need for normalization, because many parameters of supply chain models are variable (such as transport time, production capacity etc.,). The various parameters are converted to ratio and the same benchmark after fitting the relationship more representative significance.

The normalized data table was shown in Table 1.

Table 1. The Normalized Data Table

\begin{tabular}{c|c|c|c|c}
\hline Num & Ratio & $\begin{array}{l}\text { The average waiting } \\
\text { time and the initial } \\
\text { value }\end{array}$ & $\begin{array}{l}\text { The main effect of } \\
\text { average waiting time } \\
\text { and the initial value }\end{array}$ & $\begin{array}{l}\text { The residual } \\
\text { effect of its } \\
\text { initial value }\end{array}$ \\
\hline 1 & 1 & 1 & 1 & 1 \\
\hline 2 & 1.5 & 0.333333333 & 0.954368 & 0.713656 \\
\hline 3 & 2 & 0.133333333 & 1.195567 & 0.942731 \\
\hline 4 & 2.5 & 0.113333333 & 1.228162 & 0.801762 \\
\hline 5 & 3 & 0.073333333 & 0.934811 & 0.748899 \\
\hline 6 & 3.5 & 0.000733333 & 0.877445 & 0.528634 \\
\hline 7 & 4 & 0 & 0.846154 & 24.63223 \\
\hline 8 & 4.5 & 0 & 0.988266 & 685.0992 \\
\hline 9 & 5 & 0 & 0.550196 & 255.1405 \\
\hline 10 & 5.5 & 0 & 0.440678 & 204.7727 \\
\hline
\end{tabular}




\section{Conclusions}

The elasticity of supply chain risk exists in many aspects of the internal and external environment, the supply chain industry products background, upstream and downstream supply chain relationship, decision support. If not timely maintenance of the supply chain of the weak link, will pose a threat and damage the whole process of the supply chain. The construction of resilient supply chain based on core enterprise, including the establishment of supply chain information sharing mechanism, the establishment of Multi-level Supply Chain defense system and establishment of the supply chain of principal-agent mechanism and establish the supply chain emergency mechanism and so on several aspects, through information technology to improve the integration of resources together, the entire link operation efficiency, ability to achieve against the risk of supply chain.

\section{Acknowledgements}

Natural Science Foundation of Zhejiang Province ( LY16G010009 ), Education Department of Zhejiang Province(Z201120727, Y201224800), Human Resources and Social Security Bureau of Zhejiang Province(QJD1402011)

\section{References}

[1] B. D. Williams, J. Roh and T. Tokar, "Leveraging supply chain visibility for responsiveness: the moderating role of internal integration", Journal of Operations Management, vol. 31, no. 7, (2013), pp. 543-554.

[2] S. V. Guang, S. C. L. Koh and J. Baldwin, "Natural resource based green supply chain management", Supply Chain Management: An International Journal, vol. 17, no. 1, (2012), pp. 54-67.

[3] J. B. Barney, "Purchasing, supply chain management and sustained competitive advantage: The relevance of resource- based theory", Journal of Supply Chain Management, vol. 48, no. 2, (2012), pp. 3-6.

[4] C. Shepherd and H. Günter, "Measuring supply chain performance: current research and future directions", Behavioral Operations in Planning and Scheduling. Springer Berlin Heidelberg, (2011), pp. 105-121.

[5] S. Apte and B. Carlin, "Inclusion of relevant tests in the Pharmacopoeia to improve supply chain integrity", Journal of Excipients and Food Chemicals, vol. 4, no. 3, (2013), pp. 61-63.

[6] W. S. Boyce, "Supply Chain Relationships in Procurement: Is Collaboration Reality?", UNIVERSITY OF MISSOURI-SAINT LOUIS, (2014).

[7] D. Turker and C. Altuntas, "Sustainable supply chain management in the fast fashion industry: An analysis of corporate reports", European Management Journal, vol. 32, no. 5, (2014), pp. 837-849.

[8] H. Y Ching and M. A. Moreira, "Management Systems and Good Practices Related to the Sustainable Supply Chain Management", Journal of Management and Sustainability, vol. 4, no. 2, (2014), pp. 34.

[9] R. Baldwin and J. L. Gonzalez, "Supply chain Trade: A Portrait of Global Patterns and Several Testable Hypotheses", The World Economy, (2014).

[10] P. J. Daugherty, A. E. Ellinger and M. B. Myers, "Automatic Replenishment Programs: Forging Supply Chain Relationships", Proceedings of the 1999 Academy of Marketing Science (AMS) Annual Conference. Springer International Publishing, (2015), pp. 219-219.

[11] A. W. Mackelprang, J. L. Robinson and E. Bernardes, "The Relationship Between Strategic Supply Chain Integration and Performance: A Meta- Analytic Evaluation and Implications for Supply Chain Management Research", Journal of Business Logistics, vol. 35, no. 1, (2014), pp. 71-96.

[12] J. Jayaram, M. Dixit and J. Motwani, "Supply chain management capability of small and medium sized family businesses in India: A multiple case study approach", International Journal of Production Economics, vol. 147, (2014), pp. 472-485.

[13] L. Dong, H. Tao, W. Doherty and M. Young, "A Sleep Scheduling Mechanism with PSO Collaborative Evolution for Wireless Sensor Networks", International Journal of Distributed Sensor Networks, Article ID 517250, in press.

[14] K. Choi, R. Narasimhan and S. W. Kim, "Postponement strategy for international transfer of products in a global supply chain: A system dynamics examination", Journal of Operations Management, vol. 30, no. 3, (2012), pp. 167-179. 
International Journal of $u-$ and e- Service, Science and Technology Vol. 9, No. 11 (2016) 